\title{
A comparison of the accessibility of Web applications in TV and Desktop
}

\author{
Daniel Costa \\ LASIGE, Universidade de Lisboa \\ Portugal \\ dancosta@di.fc.ul.pt
}

\author{
Ricardo Dias \\ INESC-ID, Instituto Superior Técnico, \\ Universidade de Lisboa, Portugal \\ ricardo.dias@tecnico.ulisboa.pt
}

\author{
Carlos Duarte \\ LASIGE, Universidade de Lisboa \\ Portugal \\ cad@di.fc.ul.pt
}

\begin{abstract}
Given TVs reach, we envision the use of TV based applications by a wide range of the population, including the elderly and people with different kinds of impairments. Thus, it is paramount to guarantee that everyone can access the same information. In this paper we present a study of the current state of the accessibility of TV applications, analysing their conformance with the WCAG 2.0 guidelines and comparing them to their desktop versions. The results show that, even though there has been less effort in TV accessibility research, TV applications are more conformant than their desktop counterparts.
\end{abstract}

Accessibility, TV platforms, TV applications, Accessibility Guidelines, WCAG 2.0

\section{INTRODUCTION}

Connected TV platforms extend the reach of multimedia content by enabling access both to the broadcast of digital content and to Internet's multimedia content, including video-on-demand, games, social networks and much more. These platforms are becoming increasingly common, with major brands such as Apple, Google and Samsung investing in this field. As these technologies become standard it is paramount that their features are accessible by all. In this work we give special attention to TV applications - web based application software that is specifically designed for TV environments. Our goal is to evaluate the current state of accessibility of this kind of applications as we foresee an increase of the use of Connected TV platforms. Our assumption is that TV applications, lacking specific accessibility guidelines for development when compared with their desktop counterparts, are less accessible than the desktop versions. Our approach is to gather a sample of off-the-shelf TV applications that have a desktop version and perform an evaluation using a state of the art accessibility evaluation tool that follows the Web Content Accessibility Guidelines 2.0.

\section{CURRENT CONTEXT}

Existing TV Platform manufacturers usually have their own middleware, specifications, operating systems and programming languages. The unifying feature of several existing TV platforms is that they use Web technologies such as HTML5 and JavaScript in their applications. The majority of those platforms use a Browser as their runtime environment. Consequently, it can be expected that these platforms bring to the user the accessibility barriers and solutions that are currently found on classical desktop computers. Although these barriers have been extensively researched on regular Webpages, little has been done regarding Web applications directed to TV platforms.

When Web Accessibility Guidelines are mentioned, the most renowned are the World Wide Web Consortium (W3C) Web Content Accessibility Guidelines (WCAG) (Caldwell et al. 2008), which cover a wide range of recommendations for making Web content more accessible. Although the WCAG 2.0 are the standard for Web accessibility, its suitability for TV is yet to be evaluated. However, compared with WCAG 2.0, TV guidelines do not offer a means to guide the development of TV applications by following some kind of checklist or techniques. This also prevents the support for an automated evaluation. For these reasons we adopted WCAG 2.0 in our study.

\section{EXPERIMENTAL STUDY}

We performed an experimental study to understand the level of accessibility, measured by conformance to Web accessibility standards, of (Web based) applications on both platforms: TV and Desktop. The following hypothesis was defined: TV applications 
are less conformant with accessibility guidelines than their Desktop counterparts. We considered this hypothesis as the research effort and developments in the last years towards a greater accessibility of Web pages and applications was greater than that for TV applications, which are quite recent when compared to their desktop counterparts. To perform this evaluation we chose the QualWeb evaluator. This framework uses the WCAG 2.0 guidelines and it is capable of post-browsing processing evaluation (Fernandes et al. 2011). We assessed 20 applications from the Opera TV store and their respective desktop versions.

\subsection{Accessibility Analysis}

The QualWeb evaluator presents the accessibility results in terms of PASS, WARNING and FAIL. A PASS or a FAIL occurs if the technique is applicable to an HTML document and if they are in agreement or disagreement with the $\mathrm{W} 3 \mathrm{C}$ recommendations, respectively. A WARNING occurs if it is not possible to identify certain characteristic of an element as right or wrong, without the intervention of a human expert. For this study we used three metrics defined by (Lopes et al. 2010) to verify the Accessibility quality of a given webpage (in this case a Web based application): Conservative, Optimistic and Strict. Each metric yealds a value from 0 (not conformant) to 1 (fully conformant).

To check for any significant differences between the conformance in the two platforms we began by assessing the normality of the data. The results for Conservative and Optimistic metrics showed data with a normal distribution. We conducted a paired ttest for each metric comparing the conformance of the same application in both platforms. We found that there was a significant effect on conformance of the application based on the platform $(t(19)=$ $5.09, p=0.00006)$ with TV versions $(M=0.72, S D=$ $0.15)$ being more conformant than desktop $(M=$ $0.44, S D=0.15)$ versions for the Conservative metric. The Optimistic rate $(t(19)=3.68, p=0.002)$ also showed statistical differences between the TV $(M=0.87, S D=0.07)$ and Desktop $(M=$ $0.78, S D=0.05)$ versions. The normal distribution of the data on the strict rate was not verified, therefore we used a non parametric test. We conducted a Wilcoxon test and found that there was a significant effect on conformance of the application based on the platform $(K=3.21, p=0.001)$ with TV versions ( $M=0.84, S D=0.1$ ) being more conformant than desktop $(M=0.65, S D=0.12)$ versions.

\section{CONCLUSION}

The results of this experiment reject our hypothesis. The outcome revealed that TV applications are more conformant with the WCAG 2.0 accessibility guidelines than their desktop versions. One of the possible explanations for this is related with the correlation between the metric values and the page complexity. (Lopes et al. 2010) defines the complexity as the number of HTML elements present in a Web page, encompassing both the breadth and depth of the HTML node tree. They found that, at least on the conservative and strict metrics, there is an exponential decay between the node count and the metric. Our results show that in average the element count of the TV applications ( $M=$ $255, S D=194)$ is less than a fifth when compared with the desktop applications ( $M=1554, S D=$ 923). The lesser complexity of TV applications' web pages is then a probable justification for their larger conformance.

If TV applications are more conformant with accessibility guidelines, why are they not recommended for and used by people with disabilities or special needs? By manually verifying the functionality of the TV applications studied we found that presented information on TV is mostly restricted to videos and that some of the features available on the desktop versions are removed. Another contributing factor is the lack of support from assistive technologies for this new delivery context. Recommendations state that TV networks broadcast should provide Audio and Video Description. Although the description services help describing a particular show or movie, leveling in some way the enjoyment and viewing experience, they are not prepared for TV applications.

\section{ACKNOWLEDGMENTS}

This work was supported by national funds through Fundação para a Ciência e Tecnologia, under LaSIGE Strategic Project - PEstOE/EEI/UI0408/2014 and INESC-ID multiannual funding - PEst-OE/EEI/LA0021/2013. Ricardo Dias was supported by FCT, grant reference SFRH/BD/70939/2010.

\section{REFERENCES}

Caldwell, B., M. Cooper, L. G. Reid, and G. Vanderheiden (2008). Web Content Accessibility Guidelines (WCAG) 2.0. http: //www . w3.org/TR/ WCAG20/.

Fernandes, N., R. Lopes, and L. Carriço (2011). On web accessibility evaluation environments. In Proceedings of W4A'11, pp. 4:1-4:10. ACM.

Lopes, R., D. Gomes, and L. Carriço (2010). Web not for all: A large scale study of web accessibility. In Proceedings of W4A'10, pp. 10:1-10:4. ACM. 